\title{
Improved Energy and Sensory Properties of Instant Porridge Made from a Roasted Mixture of Grated Orange-Fleshed Sweet Potatoes and Flour Made from Shredded Sun Dried Cassava
}

\author{
Irene Stuart Torrie de Carvalho ${ }^{1,2^{*}}$, Lucas Daniel Tivana ${ }^{1,2}$, Yvonne Granfeldt ${ }^{1}$, \\ Petr Dejmek ${ }^{1}$ \\ ${ }^{1}$ Food Technology, Lund University, Lund, Sweden \\ ${ }^{2}$ Chemical Engineering Department, Eduardo Mondlane University, Maputo, Mozambique \\ Email: Irene.Carvalho@food.Ith.se
}

Received 8 June 2014; revised 12 July 2014; accepted 20 July 2014

Copyright (C) 2014 by authors and Scientific Research Publishing Inc.

This work is licensed under the Creative Commons Attribution International License (CC BY).

http://creativecommons.org/licenses/by/4.0/

(c) (i) Open Access

\begin{abstract}
Mozambican diets are often lacking in vitamin A. Orange-fleshed sweet potatoes (OFSP) are rich in beta-carotene, a precursor of vitamin $A$, but are not easily introduced into the diet as the consistency of the porridge made from OFSP is unappetising. Flour made from roasted cassava (garri) produces a palatable porridge with a high energy density. We propose a simple procedure for producing an instant porridge by roasting grated OFSP with flour from shredded sun-dried cassava (G-OFSP). This is an easily adopted variant of the traditional garri-making process. The consistency of this porridge made from milled G-OFSP (G-OFSPf) was compared to the consistency of porridges made from maize flour (Mf), cassava flour (Cf), OFSP flour (OFSPf) and garri flour (Gf) at $43^{\circ} \mathrm{C}$, and then correlated to the perceived characteristics assessed by a sensory panel. The dry matter concentration of the porridges was adjusted to give similar consistency, measured by the maximum force of back extrusion, using traditional maize porridge as a reference. The porridges were additionally characterized by back extrusion force relaxation time. Short relaxation times were obtained for G-OFSPf, Mf and Gf porridges and longer times for Cf and OFSPf porridges, corresponding roughly to the sensory preference. In the sensory test of consistency, G-OFSPf porridge was ranked first, followed by Mf, Gf, OFSPf and Cf. In overall sensory tests comparing G-OFSPf porridge to Gf porridge, G-OFSPf porridge was significantly preferred with regard to all sensory attributes evaluated $(p<0.01)$. The energy density of the G-OFSPf porridge was $5.6 \mathrm{~kJ} / \mathrm{ml}$; the highest of the three most preferred porridges with regard to texture. The preparation of flour from a mixture of grated OFSP and shredded sun-dried cassava improved the perceived porridge consistency, increased its energy density, and its high acceptability would increase the intake of beta-

"Corresponding author.
\end{abstract}

How to cite this paper: de Carvalho, I.S.T., Tivana, L.D., Granfeldt, Y. and Dejmek, P. (2014) Improved Energy and Sensory Properties of Instant Porridge Made from a Roasted Mixture of Grated Orange-Fleshed Sweet Potatoes and Flour Made from Shredded Sun Dried Cassava. Food and Nutrition Sciences, 5, 1430-1439. http://dx.doi.org/10.4236/fns.2014.514156 


\section{carotene.}

\section{Keywords}

\section{Cassava, Consistency, Energy Density, Garri, Orange-Fleshed Sweet Potatoes}

\section{Introduction}

In recent years, the cultivation and consumption of orange-fleshed sweet potatoes (OFSP) have increased in Africa. Since the OFSP contains higher concentration of pro-vitamin A (rich in $\beta$-carotene) their consumption may reduce vitamin A deficiency which is a common problem in this part of the world [1] [2]. OFSP are mainly eaten boiled, steamed or as a processed product such as sun-dried OFSP flour or chips. The incorporation of OFSP into common wheat-based products such chapattis and mandazis [3], bread and biscuits [4] or to prepare a soft porridge has thus been promoted. However, the porridge is not well-accepted by consumers, due to the formation of a weak porridge gel and incorporation of OFSP into the cassava-based products is still uncommon.

Cassava is an important staple food in Africa, providing a source of carbohydrates in most sub-Saharan Africa countries [5] [6]. Cassava is processed to produce many kinds of products. In West Africa, garri, made from sun-dried cassava chips and roasted shredded roots, is a well-known product [7]. Roasting is important to improve the energy density of porridge made from cereals and tubers. This modifies the starch so that it does not thicken during porridge preparation, thus requiring the addition of less water than in porridge made from the raw material [8]. Garri is processed by roasting wet shredded cassava roots on a hot $\left(95^{\circ} \mathrm{C}-100^{\circ} \mathrm{C}\right)$ metal plate, during which the starch particles become agglomerated into relatively large aggregates [9]. Shredding of cassava roots aids detoxification by allowing the enzymatic conversion of toxic cyanogenic glycosides to the volatile cyanide, which is eliminated during drying or roasting [10] [11]. Cassava roots contain $60 \%-70 \%$ water, which is reduced to $40 \%-45 \%$ by roasting. A part of water content is usually reduced by mechanical pressing or by adding sun-dried cassava flour to the wet shredded roots. During the roasting process, starch granules are gelatinized while the water is evaporated, resulting in a stable, ready-to-eat, granulated, dry pre-gelatinized product (garri) similar to couscous made from cereals.

Garri is a kind of instant food, with long shelf life and can be stored for more than a year in dry conditions [12]. Garri can be eaten dry, or reconstituted in warm or hot water or tea. It is also common for garri to be milled to flour and used to make porridge [13] [14]. Porridge made from flour produced from sun-dried cassava chips is rather rubbery in consistency and not appreciated by consumers either. The texture of porridge made from roasted cassava (garri) is more palatable, and has a higher energy density than that made from sun-dried cassava chips [14]. However, most of the cassava roots cultivated in Africa do not contain carotenoids and the incorporation of OFSP in the popular African garri may result also in porridge with a high energy density, an acceptable texture, as perceived by consumers, and could be a way to increase the intake of vitamin A.

In this study, a minor modification of the traditional method of preparing garri, by adding mixed fresh grated OFSP and flour made by shredded sun-dried cassava (G-OFSP) was evaluated. The energy density and texture of this mixture were measured, and sensory parameters such as mouthfeel, consistency, colour and flavour of an instant porridge made from the milled G-OFSP were determined.

\section{Materials and Methods}

\subsection{Samples and Sample Preparation}

The raw materials used were orange-fleshed sweet potato (Ipomoea batatas), cassava (Manihot esculenta) roots (Table 1) and maize (Zea mays) flour that was purchased from a local market.

\subsubsection{OFSP Roots}

The roots of OFSP, Erica variety, were purchased from a farm in the Boane district, in the province of Maputo, Mozambique. The roots were transported to Inharrime district, in the province of Inhambane, Mozambique, and processed $24 \mathrm{~h}$ after harvest. The OFSP roots were weighed, washed in water to remove soil, and then peeled using a knife, washed with tap water and grated $(1.0 \pm 0.5 \mathrm{~mm})$ using a motorized grater (International Institute of 
Table 1. Raw materials, processing, products and abbreviations.

\begin{tabular}{ccc}
\hline Raw material & Processing & Product \\
\hline Fresh cassava root & Peeled, shredded and sun dried & Cassava flour \\
Fresh cassava root & Peeled, shredded, pressed, roasted and milled & Garri flour \\
$\begin{array}{c}\text { Orange fleshed sweet potato } \\
\text { Cassava flour and mashed } \\
\text { orange fleshed sweet potato }\end{array}$ & Peeled, sliced, sun dried and milled & Orange fleshed sweet potato flour \\
Mix (of cassava flour with peeled and mashed & OFSPf & Garri with orange fleshed sweet \\
orange fleshed sweet potato), roasted and milled & G-OFSPf \\
\hline
\end{tabular}

Tropical Agriculture, Ibadan, Nigeria). After being grated, the material was collected and thoroughly mixed. Part of the sample was stored at $-20^{\circ} \mathrm{C}$ for analysis and remainder was used to mix with flour of shredded cassava to make G-OFSP.

\subsubsection{Cassava Roots}

Cassava roots (Xinhembwe variety) were purchased from a farm in Inharrime district, in the province of Inhambane, Mozambique. The roots were processed $2 \mathrm{~h}$ after harvesting. Cassava flour (Cf) and cassava garri flour (Gf) were prepared as described previously [9].

\subsubsection{Processing of Garri OFSP}

The G-OFSP was processed in the same way as cassava garri [9] except for a slight modification, as shown in the process scheme in Figure 1. For garri the cassava mash is pressed to decrease moisture content ahead of roasting. However, in this study the appropriate pre-roasting moisture content was achieved by addition of sun-dried cassava flour to unpressed OFSP mash. Each batch, of about $5 \mathrm{~kg}$ freshly grated OFSP, containing 70\% moisture, was thoroughly mixed with approximately $2 \mathrm{~kg}$ of flour from sun-dried shredded cassava (Cf) with 8\% moisture content, to reduce the moisture of the mixture to around $50 \%$. The mixture was roasted on a metal tray over an open fire for about 10 - $12 \mathrm{~min}$. During roasting, the material was mixed by hand with a metal scraper. The temperature during roasting was measured with a thermocouple and was about $95^{\circ} \mathrm{C}\left( \pm 1^{\circ} \mathrm{C}\right)$. After roasting, the product was left at ambient temperature $\left(30^{\circ} \mathrm{C}-33^{\circ} \mathrm{C}\right)$ for approximately $12 \mathrm{~h}$ (overnight) to cool, and to allow evaporation of some of the remaining water, resulting in a final product with about $11 \% \pm 2 \%$ moisture content. The product was then collected, thoroughly mixed and part of it was sealed in vacuum bags and stored for later analysis, and the rest was used to make porridge for sensory evaluation.

\subsubsection{Maize Flour}

Maize flour (Mf), TOP SCORE (Sasseka company, Matola, Mozambique) was purchased in Mozambique.

\subsection{Dry Matter Determination and Energy Density}

The dry matter contents of freshly grated OFSP, flour from shredded, sun-dried cassava (Cf), G-OFSP, cassava garri (Gf) and maize (Mf) were determined by drying duplicate $5 \mathrm{~g}$ samples at $105^{\circ} \mathrm{C}$ to constant weight (minimum $24 \mathrm{~h})$ according to AOAC [15].

The energy density $(\mathrm{kJ} / \mathrm{ml})$ of porridge was calculated according to the flour composition in USDA data tables [16].

\subsection{Gelatinization of Starch}

The endothermic values of the raw materials (fresh OFSP and Cf) and G-OFSPf were determined with differential scanning calorimetry (DSC) (Model 6200, Seiko Instruments, Shizuoka, Japan) using sealed aluminium pans (TA Instruments, Newcastle, USA), as described previously [9]. Samples of fresh OFSP were peeled, cut into small pieces using a knife and crushed using a mortar and pestle, and about of $6 \mathrm{mg}$ of this material was placed in the pans. The Cf and garri samples were milled in a rotor mill (Retsch ZM 1, Dusselldorf, Germany) equipped with a $0.5 \mathrm{~mm}$ mesh. A small amount of each sample (about $2 \mathrm{mg}$ ) was added to the pan and water, corresponding to about three times the weight of the sample, was added. Thermograms were recorded from $20^{\circ} \mathrm{C}$ to $120^{\circ} \mathrm{C}$ using heating and cooling rates of 10 and $20^{\circ} \mathrm{C} / \mathrm{min}$. An empty pan was used as a reference from the curve obtained, the onset temperature, peak temperature, conclusion temperature, and heat of transition were calculated automatically and the measurements were carried out three times on each sample. 


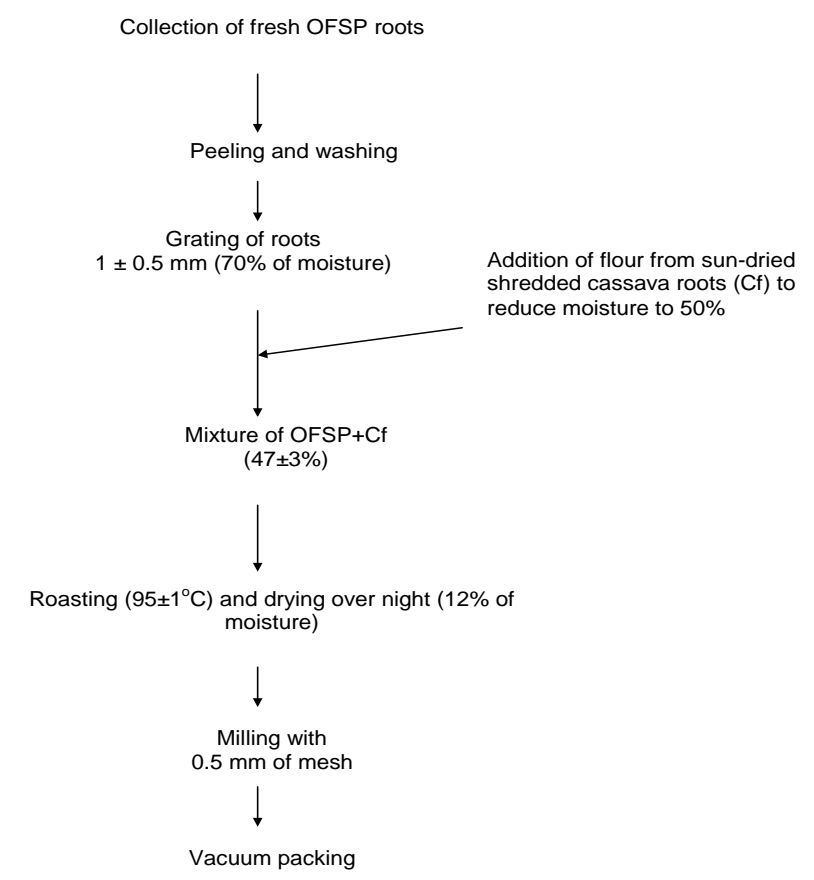

Figure 1. The preparation of the flour used to produce OFSPcomplemented cassava garri.

\subsection{Porridge Preparation}

Soft porridges were prepared from Mf, OFSPf, Cf, Gf and G-OFSPf. Maize flour (Mf) was used as a reference since it is the flour most commonly used for the preparation of soft porridge for breakfast in Mozambique. To obtain an estimate of the proportions of $\mathrm{Mf}$ and water used to make porridge, 10 members of staff at the Eduardo Mondlane University were asked to add Mf to $300 \mathrm{ml}$ of heated water in a beaker immersed in a water bath set to $100^{\circ} \mathrm{C}$ until the desired porridge consistency was achieved. The average amount of Mf added was used to make the reference porridge consistency to the other flour samples.

Different concentrations of each flour (Mf, Cf, OFSPf) were used to prepare porridges with different consistencies until similar desired consistency of maize porridge, used as reference, was achieved. Different amounts of each flour (Mf, Cf, OFSPf) were added to $40 \mathrm{ml}$ water and the samples were heated at $100^{\circ} \mathrm{C}$ for $8 \mathrm{~min}$, with constant stirring. The porridge was then cooled to a temperature of $\sim 43^{\circ} \mathrm{C}$ (approximating the normal consumption temperature). All samples were prepared and measured in triplicate.

Each of the two ready-to-use porridges (Gf and G-OFSPf) was prepared as follows: different amounts of each flour were added to $40 \mathrm{ml}$ of water at $60^{\circ} \mathrm{C}$ and held at $60^{\circ} \mathrm{C}$ for $1 \mathrm{~min}$. Afterwards, the temperature of the porridge was allowed to decrease to $\sim 43^{\circ} \mathrm{C}$ (simulating feeding temperature). All samples were prepared and measured in triplicate.

\subsection{Mechanical Characterization of the Consistency of the Porridges}

The consistency of the porridges was measured using the back-extrusion method as described previously [17] with a slight modification; namely, including a $60 \mathrm{~s}$ relaxation time after the probe had been inserted into the sample to the final depth. A texture analyser (TA.XT2i, Stable Micro Systems, Godalming, UK) was used. The measuring element was a rod with a disc $(3.0 \mathrm{~cm}$ diameter, $0.3 \mathrm{~cm}$ thickness). The measurements were performed in the beaker in which the samples were cooked and cooled to feeding temperature. The following settings were used: test speed $1.0 \mathrm{~mm} / \mathrm{s}$, trigger level $0.049 \mathrm{~N}$ and travel distance $10 \mathrm{~mm}$. Two parameters were derived when the curve force-time was recorded: porridge consistency, defined as the maximum force of back extrusion obtained as the probe penetrated the porridge, and porridge stickiness, defined as the maximum force of relaxation recorded after 10 seconds at the final depth. All measurements were performed in triplicate on three separately prepared samples. 


\subsection{Colour Measurements}

Colour measurements, based on the CIE Lab method, were performed as described previously [18], using a Konica Minolta CM-700D spectrophotometer (SENSING Inc., Japan). After calibration with a white tile, the lightness index $\left(L^{*}\right)$, red index $\left(a^{*}\right)$ and yellow index $\left(b^{*}\right)$ were recorded. The hue angle $\left(\tan ^{-1} b^{*} / a^{*}\right)$ and chroma $\left(\left(a^{* 2}+b^{* 2}\right)^{1 / 2}\right)$ were calculated from $a^{*}$ and $b^{*}$. All measurements were performed in triplicate on three separately prepared samples.

\subsection{Microscopy}

The microstructure of the porridge samples made from Mf and G-OFSPf suspended in water $\left(43^{\circ} \mathrm{C}\right)$ was observed using a light microscope (Olympus, model BX50F4, Olympus Optical Co. Ltd., Tokyo, Japan) connected to a colour video camera (model DFK41AF02, The Imaging Source Europe GmbH, Bremen, Germany). Each sample was placed on a slide and viewed at $10 \times$ magnification.

\subsection{Sensory Ranking of Consistency of Different Porridges}

Tests were carried out to compare the sensory consistency of G-OFSPf, Mf, Cf, Gf and OFSPf porridges characterized by equal back extrusion force. The panel consisted of 50 volunteers from Universidade Eduardo Mondlane (UEM), including students (about 80\%) and staff. The evaluators were asked to rank the 5 porridges from the best to the worst, with regard to the consistency sensation perceived when handling the porridge with a spoon and how easily it could be chewed in the mouth.

\subsection{Sensory Acceptability of G-OFSPf Porridge}

Sensory testing was carried out to evaluate the general acceptability of G-OFSPf porridge. The method used for the sensory tests was attribute difference tests, using hedonic rating against a reference, adapted from the work of Bergara-Almeida and da Silva [19]. An untrained panel, consisting of 84 panelists who were regular consumers of garri, was used to evaluate the G-OFSPf porridge and compare it with Gf porridge at the same consistency. These were farmers from the Josina Machel Association, local people from a rural market, and staff from the SDAE (Serviços Distritais de Actividades Económicas) in Inharrime district, in the province of Inhambane, Mozambique. The panel evaluated the appearance, odour, colour, taste, texture, sweetness and acceptability of each of the samples (Table 2). The sensory attributes were scored using a well know garri product as a reference. The samples were presented in disposable plastic cups, and unsalted crackers and water were provided to cleanse the palate between samples.

\subsection{Statistical Analysis}

The results of the acceptability of consistency were analysed with the Friedman test with significant differences at $\mathrm{p}<0.05$ and the Binomial Test was used to determine the differences of the mean scores for overall sensory acceptability and for the colour multi-comparison using Statistical Package for Science Research (SPSS), version 17.0.

\section{Results and Discussion}

\subsection{Gelatinization of Starch}

The degree of gelatinization of cassava starch in the garri provides an indication of the quality of the roasting

Table 2. Scales and scores for the various sensory attributes of G-OFSPf porridge in relation to Gf porridge.

\begin{tabular}{ccc}
\hline Appearance, odour, texture, taste and colour & Sweetness & \multicolumn{2}{c}{ Score } \\
\hline & G-OFSPf porridge in relation to Gf porridge & \\
\hline Much more preferable & Much sweeter & 5 \\
Slightly more preferable & Slightly sweeter & 4 \\
Same preference & Same sweetness & 3 \\
Slightly less preferable & Slightly less sweet \\
Much less preferable & Much less sweet & 2 \\
\hline
\end{tabular}


process, which depends on the temperature, the water content of the shredded mass, and the time until the granulated garri particles appear dried. Figure 2 shows the thermograms obtained from DSC of fresh OFSP, Cf and G-OFSPf. The Cf thermogram describes endothermic enthalpy corresponding to cassava starch gelatinization of $11.2 \pm 0.6 \mathrm{~J} / \mathrm{g}$, with a peak temperature of $71.1^{\circ} \mathrm{C} \pm 0.2^{\circ} \mathrm{C}$, an onset temperature of $58.1^{\circ} \mathrm{C} \pm 0.3^{\circ} \mathrm{C}$ and a conclusion temperature of $79.1^{\circ} \mathrm{C} \pm 0.2^{\circ} \mathrm{C}$, while fresh OFSP shows endothermic enthalpy corresponding to sweet potato starch of $10.0 \pm 0.1 \mathrm{~J} / \mathrm{g}$, with a peak temperature of $78.0^{\circ} \mathrm{C} \pm 4^{\circ} \mathrm{C}$, an onset temperature of $69.1^{\circ} \mathrm{C} \pm$ $0.5^{\circ} \mathrm{C}$ and a conclusion temperature of $85.1^{\circ} \mathrm{C} \pm 0.2^{\circ} \mathrm{C}$. The results are in agreement with those reported by Tivana et al. [9] for cassava starch, and by Waramboi et al. [20] for sweet potato starch. However, no endothermic peak was observed for G-OFSPf, indicating total gelatinization of the starch in cassava and sweet potatoes for the temperature, moisture and duration used during the preparation of the G-OFSPf. It should be emphasized that the temperature during roasting should be higher than the final temperature of the sweet potato starch gelatinization transition seen in the thermogram to ensure total gelatinization of the starch in the mixture.

\subsection{Mechanical Characterization of the Consistency of the Porridges and Energy Density}

The consistency is an important attribute for the acceptability of a porridge; and we therefore performed mechanical measurements of the consistency of other kinds of porridge, to predict the consistency that would be perceived as acceptable by consumers of the instant porridge made from G-OFSPf. The consistency of the Cf, OFSPf, Gf and Mf porridges was also measured, defined before as the maximum force obtained as the probe penetrated the porridge, and Mf porridge was used as a reference, as this is the most typical soft porridge consumed in Africa [21]. Figure 3(a) shows a typical porridge force deformation curve during back extrusion of Mf porridge. The maximum forces measured in 10 seconds of back extrusion of the different porridges with different flour concentrations are shown in Figure 3(b). Table 3 gives the energy density of the porridges based on the dry matter (DM) concentration. The DM concentration of Gf was $11 \%$ higher than that of Cf, showing that the roasting process improved the energy density of porridge comparing to sun drying cassava roots alone. G-OFSPf porridge had 1.8 times the energy density of Gf porridge, demonstrating that the addition of grated OFSP and shredded cassava almost doubled the energy density of ordinary cassava garri porridge. Maize flour had the lowest energy density and OFSPf had the highest energy density.

The stickiness measurements on the porridges were also performed, as the maximum relaxation force recorded after 10 seconds at the final depth. The relaxation force for Mf porridge is shown in Figure 3(a). Figure 4(a) gives the time taken for the maximum force to decrease by 33\%, and Figure 4(b) the corresponding values as a function of DM concentration. Only slight differences in relaxation time were observed for Cf, Gf and G-OFSPf porridges with increasing force and DM concentration, while the OFSPf behaved differently, showing an increase in relaxation time with increasing maximum force and DM concentration. The Cf porridge had a longer relaxation time than the Gf and G-OFSPf porridges, while the Gf and G-OFSPf porridges had similar relaxation times to that of Mf porridge.

\subsection{The Colour of the Flours and Porridges}

Colour is an important parameter in food processing, as it may provide information on nutrients, the freshness of the food, and the type and intensity of processing. Colour is also important for the sensory perception of food by consumers [22]. The intention of this study was to prepare a product similar to cassava garri, including OFSP which is rich in carotenoids. OFSP are characterized by their yellow-orange colour, which is perceived as an indication of a high content of carotenoids, including $\beta$-carotene, a precursor of vitamin A [23]. The OFSPf, Gf and G-OFSPf and the corresponding porridges are shown in Figure 5 and Table 4 presents the colour indices of the same materials. From Table 4 it can be seen that G-OFSPf has similar hue angle (yellow-orange colour) to OFSPf. However, the colour of G-OFSPf was more intense (higher chroma value) than that of OFSPf. G-OFSPf was less light than OFSPf. The hue angle and chroma of G-OFSPf may indicate that there is significant retention of the colour from OFSP during roasting. The higher value of chroma for G-OFSPf than for OFSPf may also be due to the Maillard reaction during the roasting process.

Comparing the porridges made from the flours, the hue angle of the porridges made from OFSPf and G-OFSPf are similar and the yellow-orange colour was preserved. OFSPf porridge had a significantly higher chroma value than the porridge made from G-OFSPf. Porridge made from Gf had the greatest lightness, the lowest chroma value, and its hue angle was outside the yellow-orange region, and showed a very low intensity green-blue hue. 


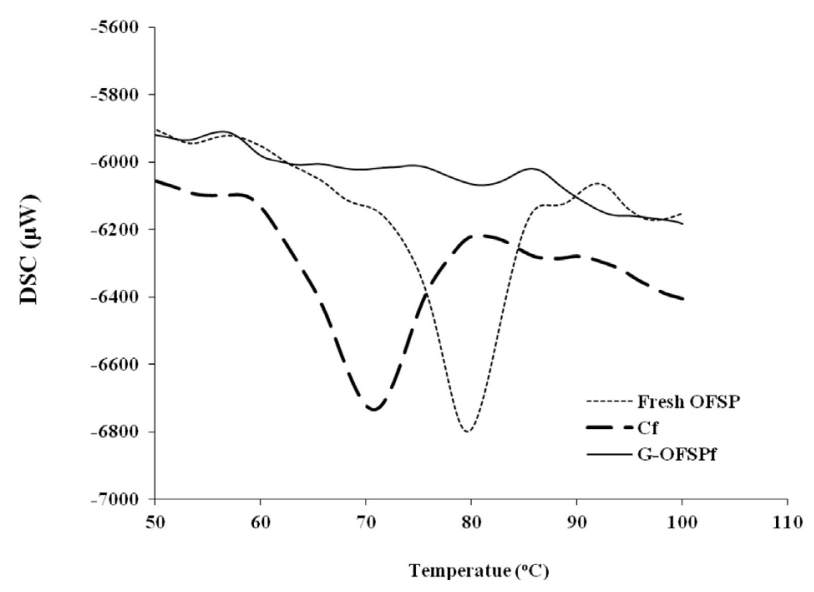

Figure 2. The thermograms obtained from fresh OFSP, Cf and G-OFSPf, using DSC.

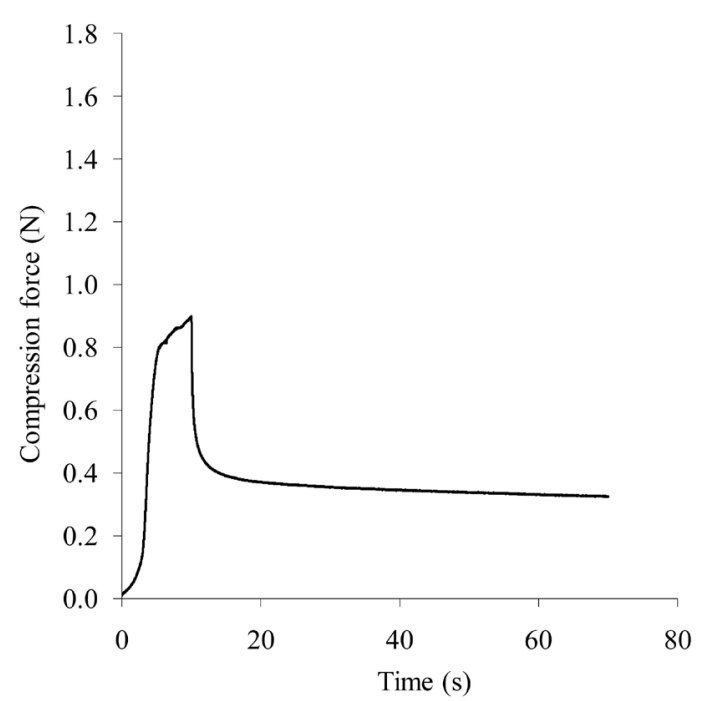

(a)

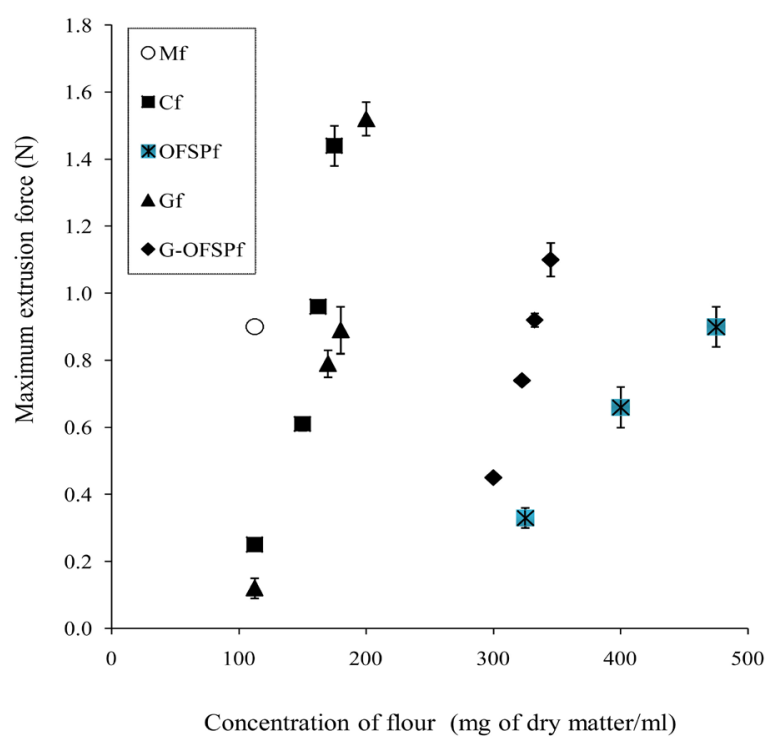

(b)

Figure 3. (a) A typical deformation curve obtained during back extrusion of maize porridge; (b) The maximum forces of the different porridges with different flour concentrations (achieved in 10 seconds of back extrusion). The error bars correspond to the standard deviation obtained from 3 independent samples.

Table 3. Dry matter and energy density of different porridges formulated to give approximately the same maximum force in back extrusion.

\begin{tabular}{ccccc}
\hline Porridge sample & Dry matter $(\mathrm{mg} / \mathrm{ml})$ & Maximum force $(\mathrm{N})$ & $\begin{array}{c}\text { Relaxation time (s) for 33\% } \\
\text { decrease in maximum force }\end{array}$ & Energy density (kJ/ml) \\
\hline Mf & 112 & 0.89 & 0.3 & 2.10 \\
Cf & 162 & 0.91 & 0.7 & 2.73 \\
OFSPf & 475 & 0.88 & 1.3 & 8.06 \\
Gf & 180 & 0.89 & 0.3 & 3.06 \\
G-OFSPf & 332 & 0.92 & 0.3 & 5.60 \\
\hline
\end{tabular}

From the results of the colour analysis, it can be concluded that the yellow-orange colour of OFSP is largely preserved during roasting of the mixture of grated fresh OFSP roots and flour from shredded sun-dried cassava. In agreement with the colour retention, our preliminary data suggest that carotenoids are preserved in G-OFSPf. 


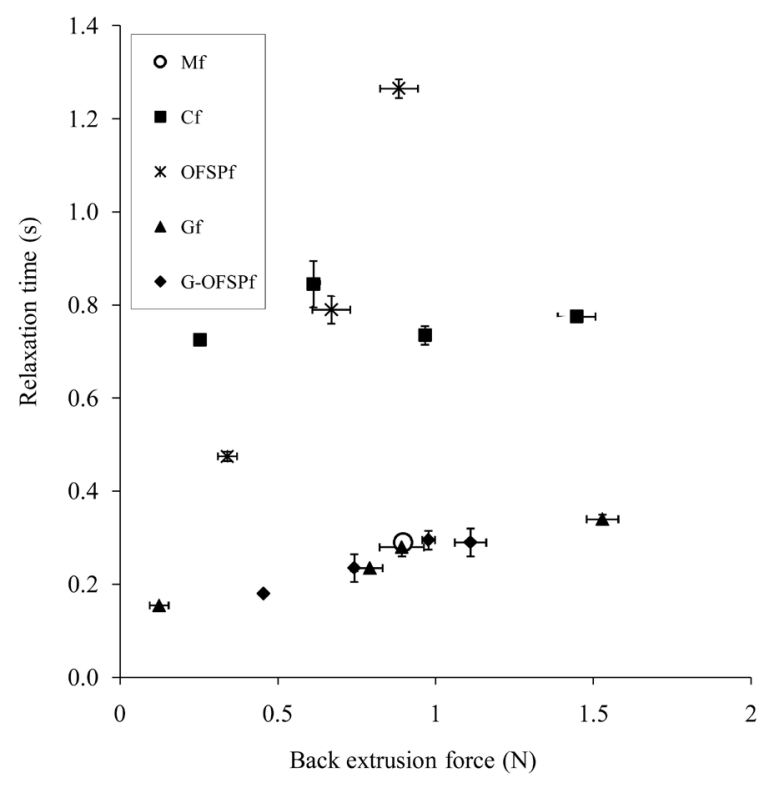

(a)

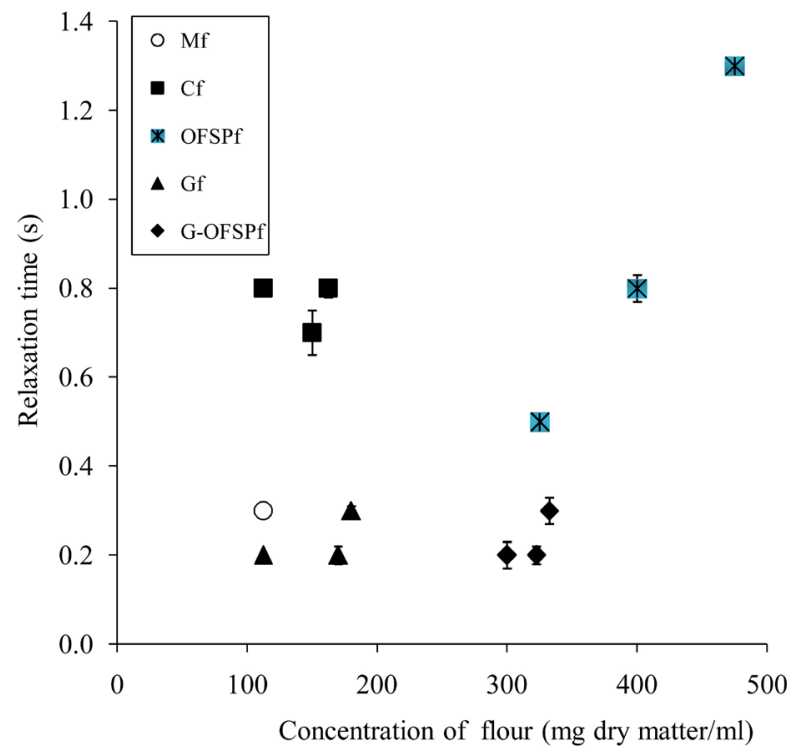

(b)

Figure 4. (a) The time required for relaxation as a function of back-extrusion force (the error bars correspond to the standard deviation obtained from 3 independent samples); (b) The relaxation time as a function of DM content (the error bars correspond to the standard deviation obtained from 3 independent samples).

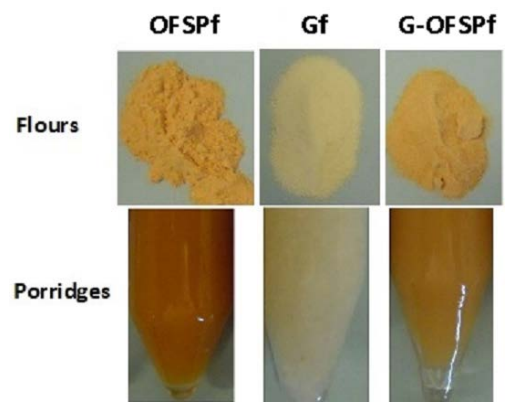

Figure 5. The colours of OFSPf, Gf and G-OFSPf and the corresponding porridges.

Table 4. Colour indices of OFSPf, Gf and G-OFSPf and the corresponding porridges.

\begin{tabular}{ccccc}
\hline & & $\mathrm{L}^{*}$ & Chroma & Hue angle $\left(^{\circ}\right)$ \\
\hline \multirow{3}{*}{ Flours } & OFSPf & $81 \pm 0^{\mathrm{a}}$ & $31 \pm 0^{\mathrm{a}}$ & $64 \pm 1^{\mathrm{a}}$ \\
& Gf & $92 \pm 0^{\mathrm{b}}$ & $13 \pm 1^{\mathrm{b}}$ & $96 \pm 1^{\mathrm{b}}$ \\
& G-OFSPf & $71 \pm 1^{\mathrm{c}}$ & $39 \pm 0^{\mathrm{c}}$ & $62 \pm 0^{\mathrm{a}}$ \\
\multirow{3}{*}{ Porridges } & OFSPf & $40 \pm 0^{\mathrm{a}}$ & $45 \pm 1^{\mathrm{a}}$ & $64 \pm 0^{\mathrm{a}}$ \\
& Gf & $60 \pm 1^{\mathrm{b}}$ & $3 \pm 0^{\mathrm{b}}$ & $147 \pm 4^{\mathrm{b}}$ \\
& G-OFSPf & $48 \pm 1^{\mathrm{c}}$ & $24 \pm 1^{\mathrm{c}}$ & $64 \pm 1^{\mathrm{a}}$ \\
\hline
\end{tabular}

Values in the same column and in same group of samples (flours or porridges) not sharing the same letters (a, b, c) are significantly different ( $<<$ 0.05).

\subsection{Microscopy Observations}

To better understand the microstructure of the porridges, they were observed under a light microscope. In Figure 6 representative images of Mf and G-OFSPf porridges are shown. The microstructure of the different porridges varied according to the material used. The images shown in the figure have a similar DM concentration (112 mg/ml). It can be observed that the Mf porridge (Figure 6(a)) contained dispersed densely packed particles. The image of porridge made from G-OFSPf (Figure 6(b)) shows a much less densely packed system of 

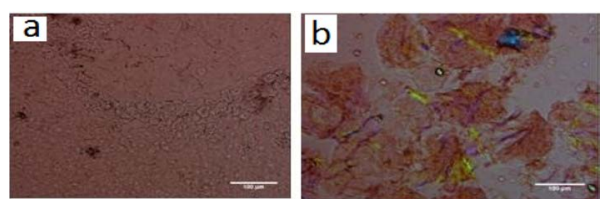

Figure 6. Microscope images of (a) Mf porridge and (b) GOFSPf porridge with similar flour concentrations (the scale bars correspond to $100 \mu \mathrm{m})$.

Table 5. Results for all sensory attributes comparing G-OFSPf porridge and Gf porridge.

\begin{tabular}{ccccccc}
\hline & & Category $^{\mathrm{d}}$ & $\mathrm{N}^{\mathrm{e}}$ & Observed Prop. $^{\mathrm{f}}$ & Test Prop. $^{\mathrm{g}}$ & Asymp. Sig. (2-tailed) $^{\text {Appearance, taste, }}$ \\
texture, & Group 1 $^{\mathrm{b}}$ & $\leq 3$ & $\leq 10$ & $\leq 0.12$ & 0.5 & $0.000^{\mathrm{a}}$ \\
sweetness & Group 2 $^{\mathrm{c}}$ & $>3$ & $\geq 74$ & $\geq 0.88$ & & \\
& Total & & 84 & 1.00 & $0.000^{\mathrm{a}}$ \\
Odour, colour, con- & Group 1 $^{\mathrm{b}}$ & $\leq 3$ & $11-17$ & $0.13-0.20$ & 0.5 & \\
sistency & Group 2 & $>3$ & $67-73$ & $0.80-0.87$ & & \\
& Total & & 84 & 1.00 & & \\
\hline
\end{tabular}

${ }^{\mathrm{a}}$ Based on Z approximation. ${ }^{\mathrm{b}}$ Group of panelists (acceptability score $\leq 3$ ). ${ }^{\mathrm{c}}$ Group of panelists (acceptability score $>3$ ). ${ }^{\mathrm{d}}$ Acceptability score. ${ }^{\mathrm{e}}$ Number of panelists. ${ }^{\mathrm{f}}$ Observed proportion in the groups. ${ }^{\mathrm{g}} \mathrm{Tested}$ proportion (50\%).

particles consisting of partially swollen agglomerates of starch granules. This is the reason for the much thinner consistency of the G-OFSPf porridge at the same DM concentration. The "open" spaces allow additional DM up to $332 \mathrm{mg} / \mathrm{ml}$ to be incorporated to achieve an acceptable consistency, similar to that of Mf porridge.

\subsection{Sensory Ranking of Consistency of Different Porridges}

To assess the perceived consistency of the porridges made from the 5 samples of flour (Mf, Cf, OFSPf, Gf, G-OFSPf), 50 panelists were asked to rank the samples from the best to the worst, according to the consistencysensation perceived on the spoon touch and breakup of the porridge and how easily can be chewed in the mouth. About $60 \%$ of the panelists ranked the G-OFSPf porridge as their first choice, followed by Gf (20\% of the panelists). In comparative rank sensory test of the consistency, using Friedman test the G-OFSPf porridge was ranked best, followed by Gf, Mf, OFSPf and Cf porridge, with G-OFSPf porridge being significantly the best appreciated of all samples $(\mathrm{p}<0.001)$.

\subsection{Overall Sensory Acceptability of G-OFSPf Porridge}

The general acceptability of G-OFSPf porridge was based on the scores of the different attributes of G-OFSPf porridge in relation to Gf porridge, given in Table 5. The evaluators rated all the sensory attributes slightly higher for G-OFSPf porridge than the Gf porridge. This study showed that the possibility exists for the use of G-OFSPf porridge as palatable and acceptable product by the consumers.

\section{Conclusion}

We have developed a simple procedure for the incorporation of OFSP into cassava garri, in order to improve its energy density and vitamin A content. The procedure is an easily adopted variant of the traditional garri making process common among low-income farmers. Roasting the cassava provides porridge with a higher energy density than sun-dried cassava flour. This was further improved by including fresh OFSP, which almost doubled theenergy density compared to normal cassava garri. Furthermore, the addition of fresh OFSP that led to yellow-orange colour porridge may be indicating increased content of $\beta$-carotene, retained even after roasting. Thus, further investigations are being carried out to evaluate the retention of $\beta$-carotene after roasting processing. Importantly, the OFSP-complemented porridge was well-accepted by local cassava garri consumers.

\section{Acknowledgements}

This study was supported by the Swedish International Development Cooperation Agency (SIDA). 


\section{References}

[1] Burri, B.J. (2011) Evaluation Sweet Potato as an Intervention Food to Prevent Vitamin A Deficiency. Comprehensive Reviews in Food Science and Food Safety, 10, 118-130. http://dx.doi.org/10.1111/j.1541-4337.2010.00146.x

[2] Low, J.W., Arimond, M., Osman, N., Cunguara, B., Zano, F. and Tschirley, D. (2007) A Food-Based Approach Introducing Orange-Fleshed Sweet Potatoes Increased Vitamin A Intanke and Serum Retinol Concentrations in Young Children in Rural Mozambique. The Journal of Nutrition, 137, 1320-1327.

[3] Bechoff, A., Poulaert, M., Tomlins, K.I., Westby, A., Menya, G., Young, S. and Dhuique-Mayer, C. (2011) Retention and Bioaccessibility of $\beta$-Carotene in Blended Foods Containing Orange-Fleshed Sweet Potato Flour. Journal of Agriculture and Food Chemistry, 59, 10373-10380. http://dx.doi.org/10.1021/jf201205y

[4] Low, J.W. and Van Jaarsveld, P.J. (2008) The Potential Contribution of Bread Buns Fortified with Beta-Carotene-Rich Sweet Potato in Central Mozambiqu E. Food and Nutrition Bulletin, 29, 98-107.

[5] Ukwuru, M.U. and Egbonu, S.E. (2013) Recent Development in Cassava-Based Products Research. Academia Journal of Food Research, 1, 001-013.

[6] Nweke, F.I., Spencer, D.S.C. and Lyanam, J.K. (2002) The Cassava Transformation. Africa’s Best Kept Secret, Michigan State University Press, East Lansing.

[7] Westby, A. (2002) Cassava Utilization, Storage and Small-Scale Processing. In: Hillocks, R.J., Thresh, J.M. and Bellotti, A.C., Eds., Cassava Biology, Production and Utilization, CABI Publishing, Wallingford, 281-300. http://dx.doi.org/10.1079/9780851995243.0281

[8] Ashworth, A. and Draper, A. (1992) The Potential of Traditional Technologies for Increasing the Energy Density of Weaning Foods: A Critical Review of Existing Knowledge with Particular Reference to Malting and Fermentation. CDD/EDP/WHO.

[9] Tivana, L.D., Dejmek, P. and Bergenståhl, B. (2010) Characterization of the Agglomeration of Roasted Shredded Cassava (Manihot esculenta Crantz) Roots. Starch/Stärke Journal, 62, 637-646.

[10] Tivana, L., Francisco, J.C., Bergenståhl, B. and Dejmek, P. (2009) Cyanogenic Potential of Roasted Cassava (Manihot esculenta Crantz) Roots Rale from Inhambane Province, Mozambique. Czech Journal of Food Sciences, 27, 375-378.

[11] Westby, A. and Choo, B.K. (1994) Cyanogen Reduction during Lactic Fermentation of Cassava. Acta Horticulturae, 375, 209-215.

[12] Meuser, F. and Smolnik, H.D. (1980) Processing of Cassava to Gari and Other Foodstuffs. Starch-Stärke Journal, 4, 116-122. http://dx.doi.org/10.1002/star.19800320405

[13] Ogundele, S.O. (2007) Understanding Aspects of Yoruba Gastronomic Culture. Indian Journal of Traditional Knowledge, 6, 50-56.

[14] Ayankunbi, M.A., Keshinro, O.O. and Egele, P. (1991) Effect of Methods of Preparation on the Nutrient Composition of Some Cassava Products-Garri (eba), "Lafun” and "Fufu”. Food Chemistry, 41, 349-354. http://dx.doi.org/10.1016/0308-8146(91)90059-W

[15] AOAC (1984) Official Methods of Analysis of the Association of Official Analytical Chemists. The Association of Official Analytical Chemists, Inc., Arlington.

[16] USDA (2013). http://ndb.nal.usda.gov/

[17] De Carvalho, I.S.T., Granfeldt, Y., Eliasson, A.C. and Dejmek, P. (2013) Predictability of the Consistency of Porridges Using Different Methods to Measure Flour Swelling. Starch-Stärke, 65, 199-207.

[18] Tivana, L.D., Dejmek, P. and Bergenståhl, B. (2013) Effect of pH and Soybean Flour Heat Treatment on the Texture and Colour of Fortified Roasted Shredded Cassava Roots (Garri). Starch-Stärke, 65, 628-636.

[19] Bergara-Almeida, S., Aparecida, M. and Da Silva, M.A.A.P. (2002) Hedonic Scale with Reference: Performance in Obtaining Predictive Models. Food Quality and Reference, 13, 57-64. http://dx.doi.org/10.1016/S0950-3293(01)00057-X

[20] Waramboi, J.G., Dennien, S., Gidley, M.J. and Sopade, P.A. (2011) Characterisation of Sweetpotato from Papua New Guinea and Australia: Physicochemical, Pasting and Gelatinisation Properties. Food Chemistry, 126, 1759-1770. http://dx.doi.org/10.1016/j.foodchem.2010.12.077

[21] De Groote, H. and Kimenju, S.C. (2012) Consumer Preferences for Maize Products in Urban Kenya. Food and Nutrition Bulletin, 33, 99-110.

[22] Coultate, T.P. (2009) Food: The Chemistry of Its Components. 5th Edition, Royal Society of Chemistry.

[23] Hagenimana, V., Carey, E.E., Gichuki, S.T., Oyunga, M.A. and Imungi, J.K. (1998) Carotenoid Contents in Fresh, Dried and Processed Sweetpotato Products. Ecology of Food and Nutrition, 37, 455-473.

http://dx.doi.org/10.1080/03670244.1998.9991560 
Scientific Research Publishing (SCIRP) is one of the largest Open Access journal publishers. It is currently publishing more than 200 open access, online, peer-reviewed journals covering a wide range of academic disciplines. SCIRP serves the worldwide academic communities and contributes to the progress and application of science with its publication.

Other selected journals from SCIRP are listed as below. Submit your manuscript to us via either submit@scirp.org or Online Submission Portal.
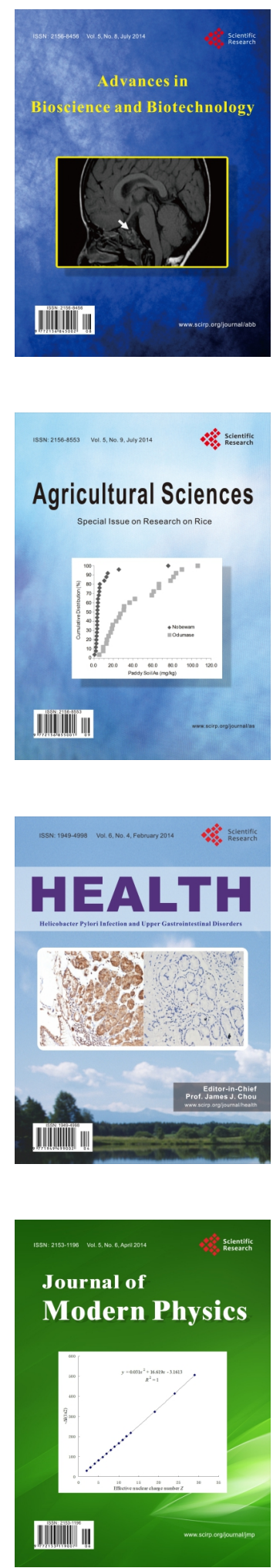
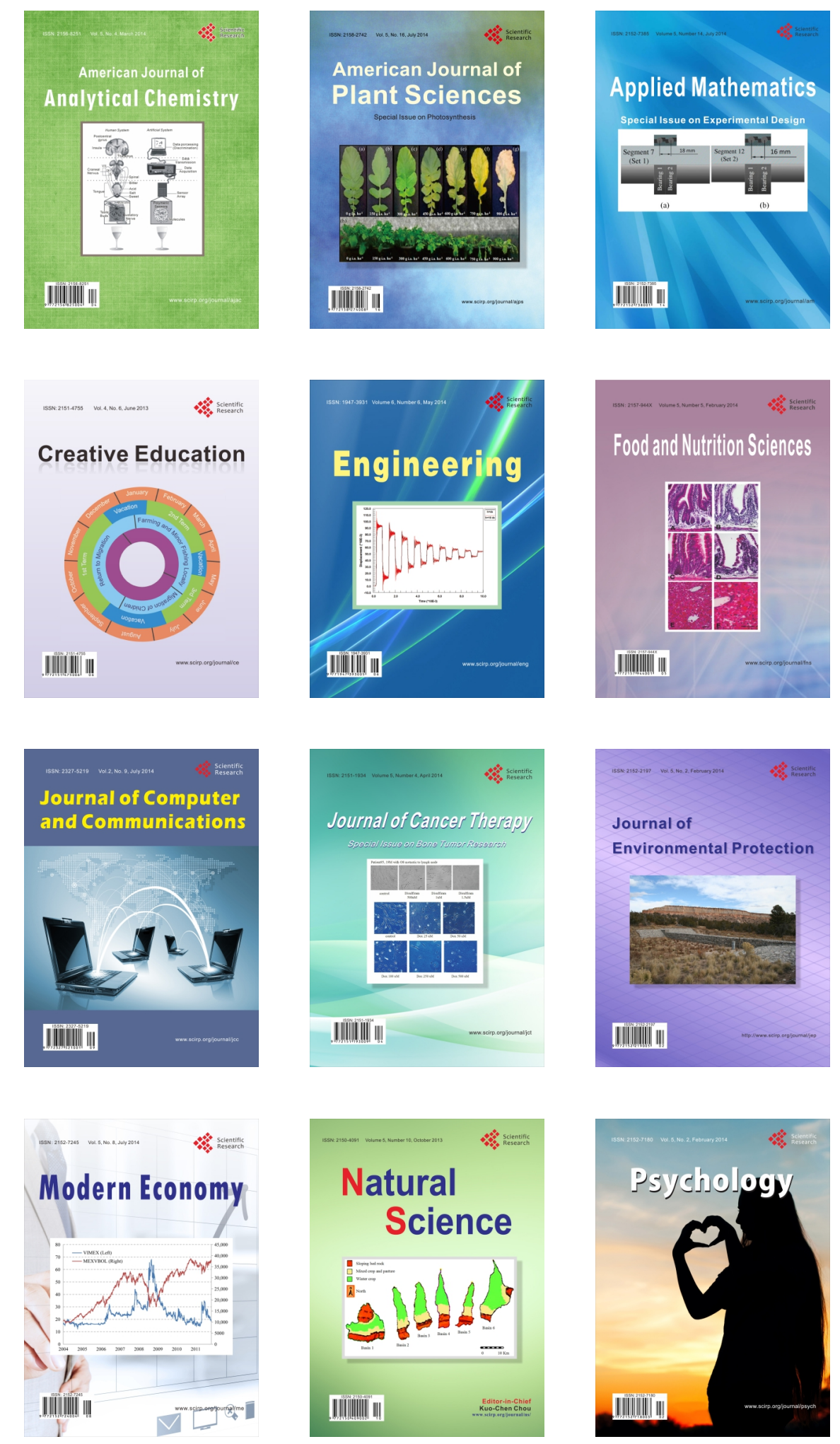